\title{
Investigate Oblique Incident Alpha Particle Tracks on CR-39 Detector
}

\author{
Yasser T. Khlile ${ }^{1 *}$, Mushtaq A. D. Al-Jubbori² \\ ${ }^{1,2}$ Department of Physics, College of Education for Pure Sciences, University of Mosul, Mosul, Iraq \\ E-mail: ${ }^{1 *}$ yasrth757@gmail.com, ${ }^{2}$ mushtaq_phy@uomosul.edu.iq
}

(Received June 05, 2020; Accepted July 05, 2020; Available online September 01, 2020)

DOI: 10.33899/edusj.2020.127268.1081, (c) 2020, College of Education for Pure Science, University of Mosul.

This is an open access article under the CC BY 4.0 license (http://creativecommons.org/licenses/by/4.0/).

\begin{abstract}
:
In this paper, the nuclear track detector CR-39 with a thickness of $200 \mu \mathrm{m}$ is divided to several pieces. The area of one piece is $1 \times 1 \mathrm{~cm}^{2}$. This study has involved two parts. Fist part is without irradiated by alpha particles. The second is irradiated by alpha particles with energy $\mathrm{E}=1.2 \mathrm{MeV}$ using the ${ }^{241} \mathrm{Am}$ radioactivity source and incident angles $(0,10,30,50)^{\circ}$. All samples etching with sodium hydroxide solution at a concentration of $6.25 \mathrm{~N}$ and a temperature of $(70 \pm 1){ }^{\circ} \mathrm{C}$. The study included calculating track parameters and comparing them with the results of the first equation from the Track-Test program. This comparison showed that there is a good agreement between experimental results and Track-Test data for track profile.
\end{abstract}

Keywords: CR-39 detector; Oblique Incident; Alpha particles; Track lengths

$$
\begin{aligned}
& \text { لدراسة آثار جسيمات الفا في حالة السقوط المائل على كاشف الأثر النووي CR-39 } \\
& \text { ياسر طه خليل1"، مشتاق عبد داود الجبوري2 } \\
& \text { 1و2قسم الفيزياء، كلية التربية للعلوم الصرفة، جامعة الموصل، الموصل، العرلق }
\end{aligned}
$$

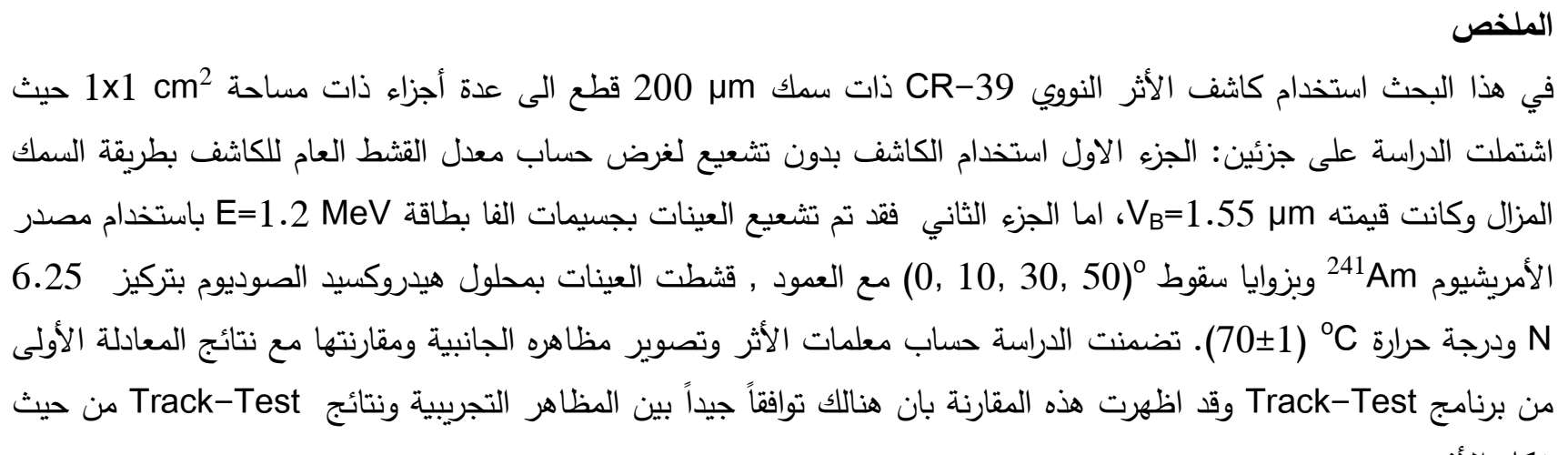
شكل الأثر.

الكلمات المفتاحية: كاشف CR-39، السقوط المائل، جسيمات الفا، اطوال الاثار. 
تمتاز كواشف الأثر النووي الصلبة بخصائص عديدة جعلت منها أداة فعالة في الكشف عن الجسيمات المشحونة وشظايا الانشطار وكذلك النيترونات [1]. من هذه الخصائص؛ وفرتها وكلفتها المناسبة، وسهولة استعمالها وعدم حاجتها إلى أجهزة الكترونية ومجهزات قدرة مما يعطي مرونة كبيرة في استعمالها في مناطق لا يمكن إيصال الأجهزة الاككترونية والمستلزمات الأخرى التي تحتاجها الكواشف الاككترونيـة. ومن الخصائص الأخرى لهذه الكواشف إن لها استجابة وكفاءة وتمتاز بدرجة كبيرة من تجانس مادتها وانتظامها، وشفافيتها البصرية العالية فضلا عن سهولة اظهار آثار الجسيمات المشحونة فيها لوفرة

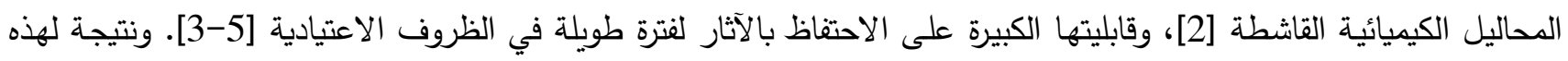
الخصائص العامة والخصائص الأخرى الإضافية التي يمتاز بها كل كاشف من كواشف الأثر النووي الصلبة تبعا لنوعه وتركيبه

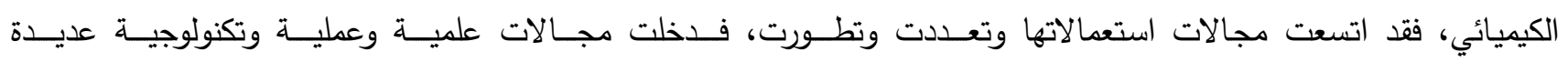
ولاسـيما فـي العقدين الأخيرين [6]؛ وهكذا فإنها تستعمل في تحديد أعمار المناطق الجيولوجية والصخور وفي علم الآثار وفي الكشف عن الجسيمات المشحونة الثقيلة كشظايا الانشطار النووي وامكانية تمييزها عن الجسيمات الأخف منها وفي تحديد تراكيز الرادون واليورانيوم في المواد والمياه والتربة وفي التتبؤ بحدوث الزلازل بقياس التغير في تراكيز الرادون المنبعث من

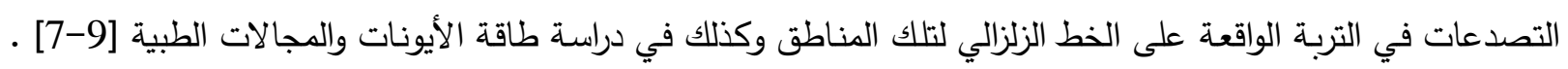
هنالك عدد من المعلمات الاساسية في عملية تكوين الاثر واظهاره نتيجة سقوط جسيمات الفا على الكاشف

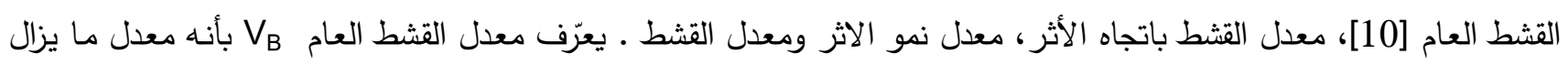
من المادة من سطح الكاشف في وحدة الزمن بفعل القشط الكيميائي للكاشف [11]. فقد يحدث تحلل تدريجي للجزيئات

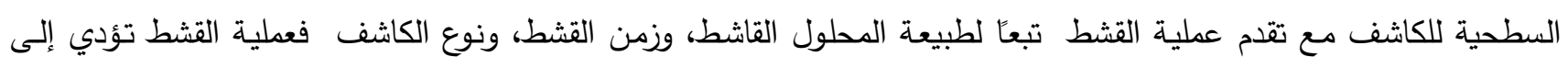

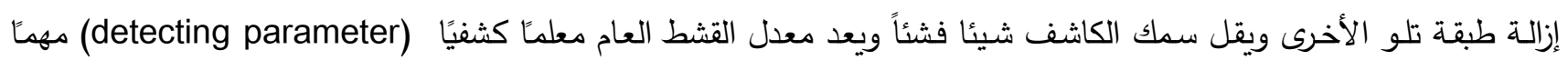
[12]، حيث يعتمد على نوع الكاشف ومنشئه ودرجـة بلمرته، ونقاوة المـادة المصنع منها، ومدى تجانس مادة الكاشف وتماثل خواصـه. اما معدل قثط الأثر(Track etching rate, VT) على طول عمق الأثر من أحد أهم امالقشطية والكثفية في كواشف الأثر

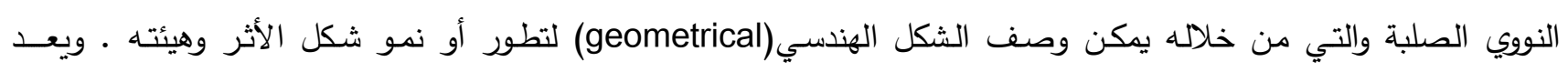

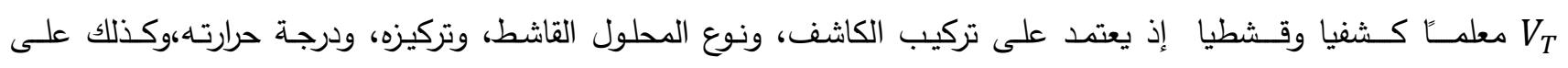

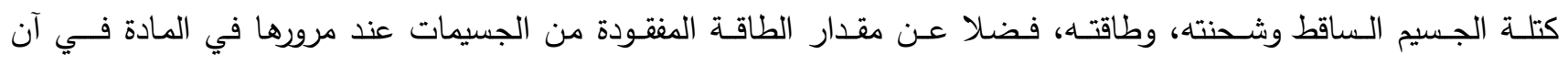

واحتد [احس [15-13].

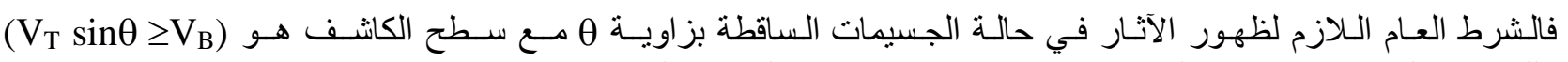

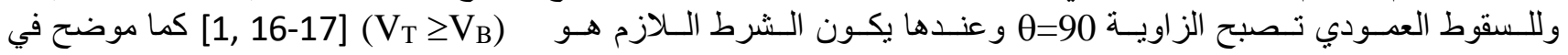

(1) الثكل لغرض دراسة أشكال الآثار المتكونة على سطح كاشف الاثر النووي CR-39 وضعت عدد من النموذجات منها أنموذج فرووم وجماعته [16] وأنموذج نيكيزيك و يوو [12] واخيرا انموذج عزوز وجماعته [9].

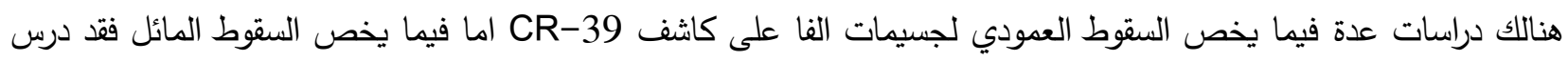
دوشيل وجماعته [1] اثار سقوط ايونات الليثيوم بطاقة 9.9MeV وجسيمات الفا بطاقات (5.9, 6.33)MeV) وبزوايا سقطوط صفر و 40 درجة مع العمود على السطح ودرس هيرمسدورف اقطار اثار جسيمات الفا وايونات الليثيوم لعدد من الطاقات وزوايا

السقوط [18]. 


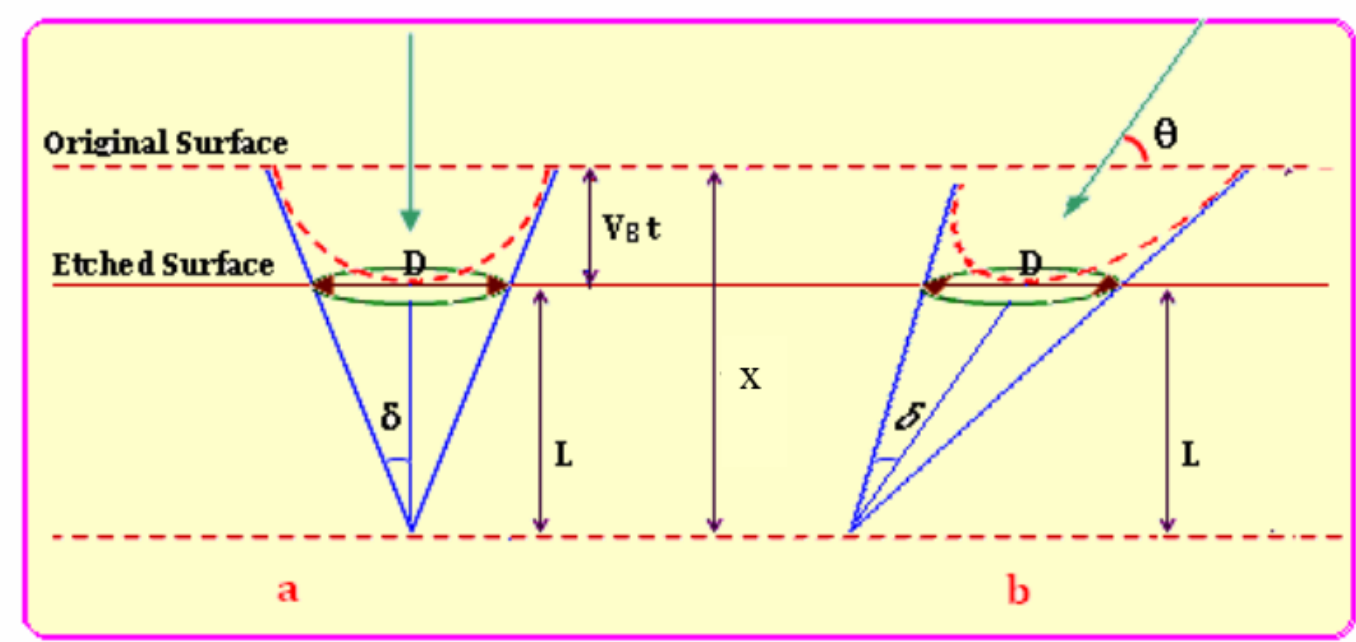

الثكل (1): شكل الأثر المقشوط للجسيم المشحون في الكاشف

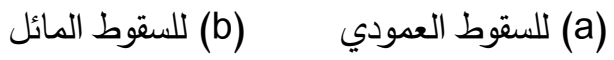

طريقة العمل

في هذا البحث تقسم طريقة العمل الى جزئين: الجزء الاول يتعلق بحساب معدل القشط العام VB للكاشف حيث استخدم كاشف لاشف

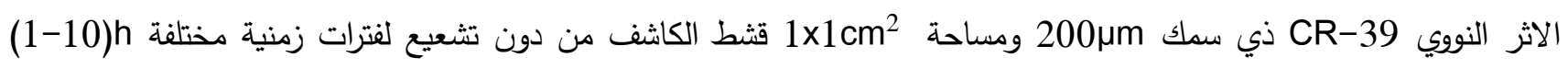
وبفترات قشطية تتابعية 30 دقيقة. اما الجزء الثاني فقد اشتمل على اخذ ثلاث عينات من الكاشف بنفس المساحة المذكورة اعلاه

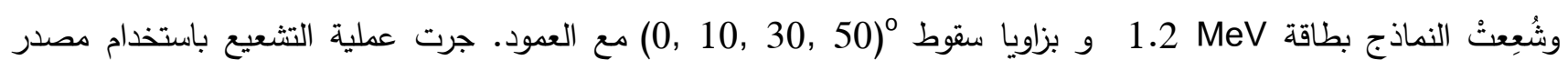
الامريشيوم Am الهواء 4.16cm ومن خلال تغيير المسافة بين المصدر والكاشف يتم الحصول على الطاقة المطلوبة. استخدم محلول هيدروكسيد الصوديوم ولحصول على صور للأثار المقشوطة فقد تم ربط كاميرا رقمية نوع MDCE-5A مع المجهر البصري المعروف بالمجهر البايولوجي (XSZ-H Series Biological Microscope)

\section{النتائج والمناقشة}

لحساب معدل القشط العام (Bulk etch rate, VB قشط الكاشف بمحلول هيدروكسيد الصوديوم بعيارية 6.25N ودرجة

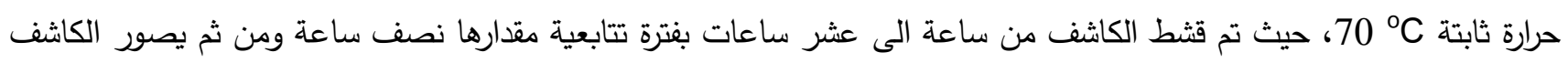

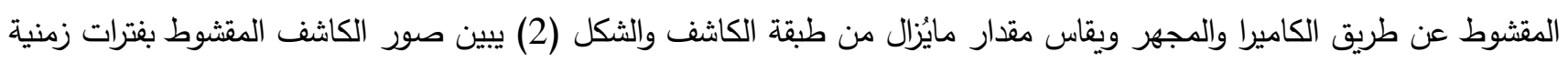




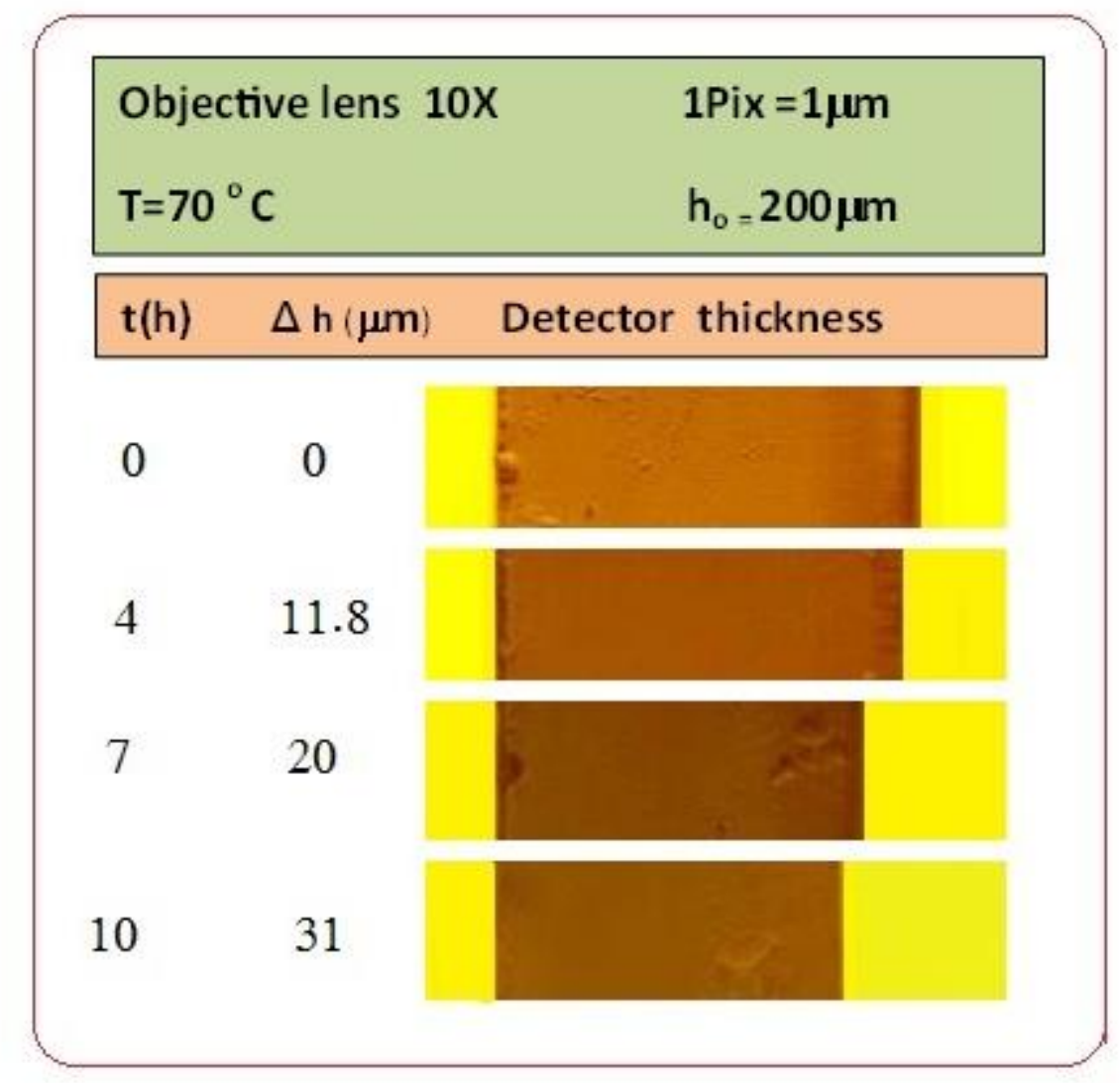

الثكل (2): صورة توضح التغير في سمك الكاشف CR-39 لأزمان قثط مختلفة

استخدمت طريقة السمك المزال لحساب VB من خلال المعادلة التالية [15].

$V_{B}=\frac{1}{2} \frac{\Delta \mathrm{h}}{\Delta t}$

(1)

هt هيث ان Dh سك الطبقة المزالة من الكاشف خلال فترة قشطية

اما معدل قشط الاثر فيمكن حسابه من المعادلة [1].

$V_{T}=\frac{1}{\cos \theta}\left[\frac{d L}{d t}+V_{B}\right]$

$V=\frac{V_{T}}{V_{B}}$

ونسبة معدل القشط يمكن حسابها من العلاقة التالية [1].

الثكل (3) يمثل العلاقة بين الطبقة المزالة من الكاشف وزمن القشط نلاحظ من الثكل ان العلاقة تكون خطية وبأخذ معدل البيانات

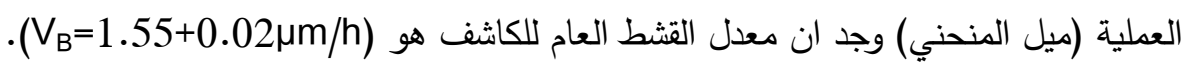




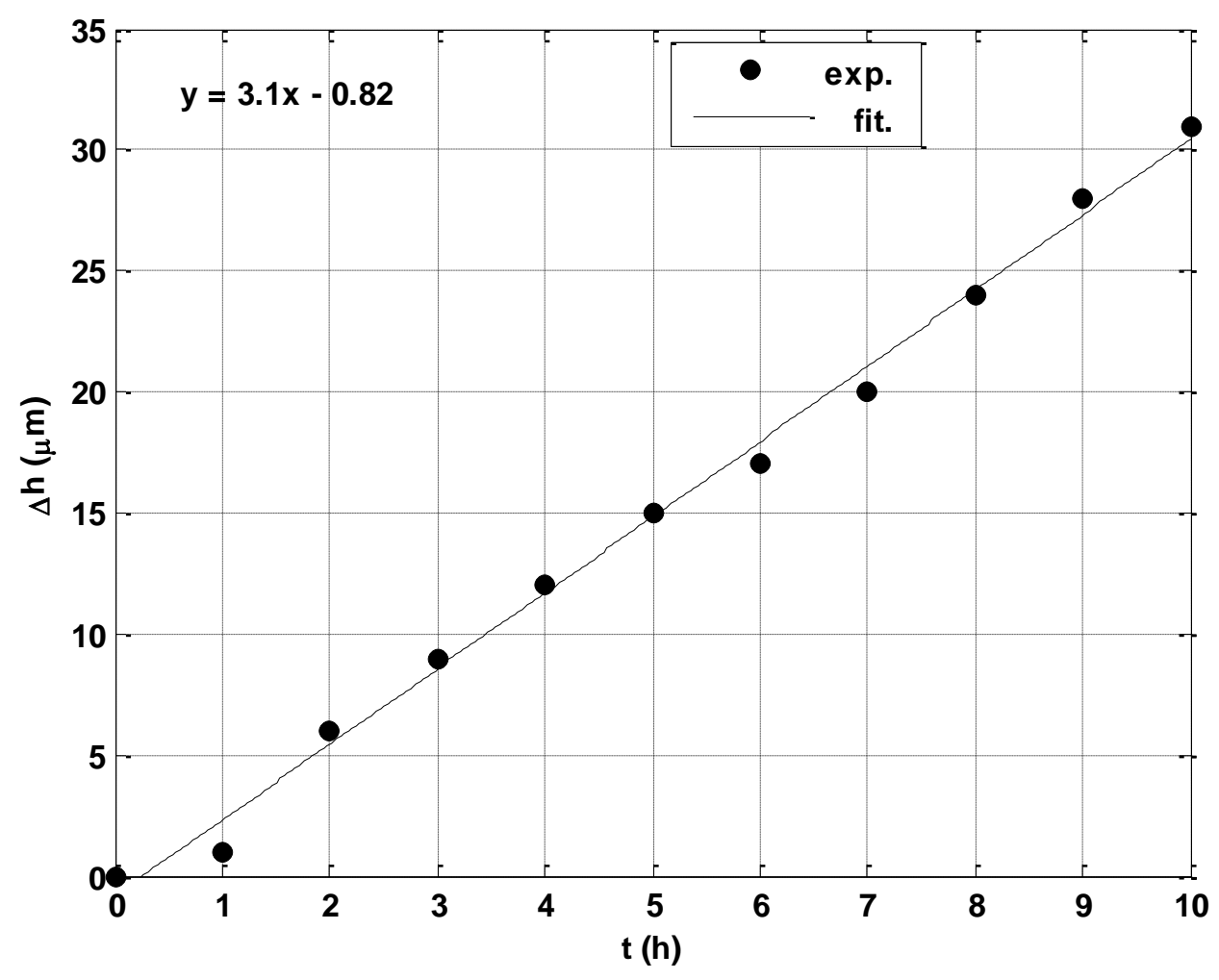

الثكل (3): الطبقة المزالة من الكاشف دالة لزمن القشط.

تم تصوير الاثار المقشوطة وقياس طولها بصورة عمودية عند كل زمن قثط والثكل (4) يمثل العلاقة بين طول الاثر دالة لزمن

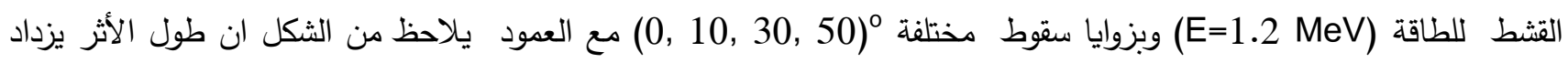

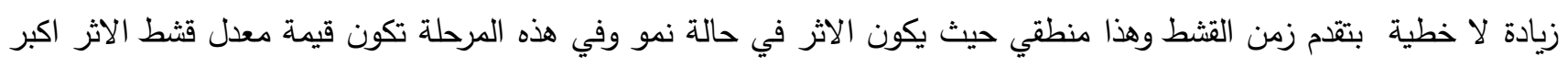

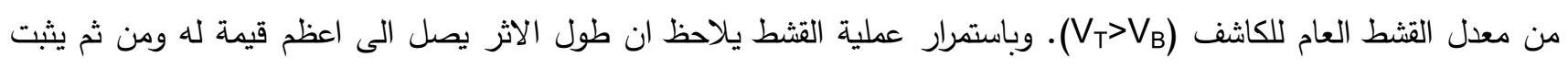
باستمرار عملية القشط وتسمى هذه المرحلة بمرحلة القشط المفرط والتي يكون عندها معدل قثط الاثر مساويا لمعدل القشط العام للكاثف (V V

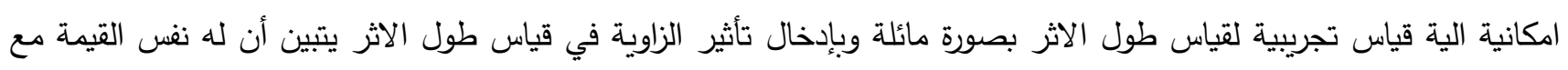
زمن القشط بغض النظر عن زاوية السقوط كما في الثكل (5) حيث النية ان 


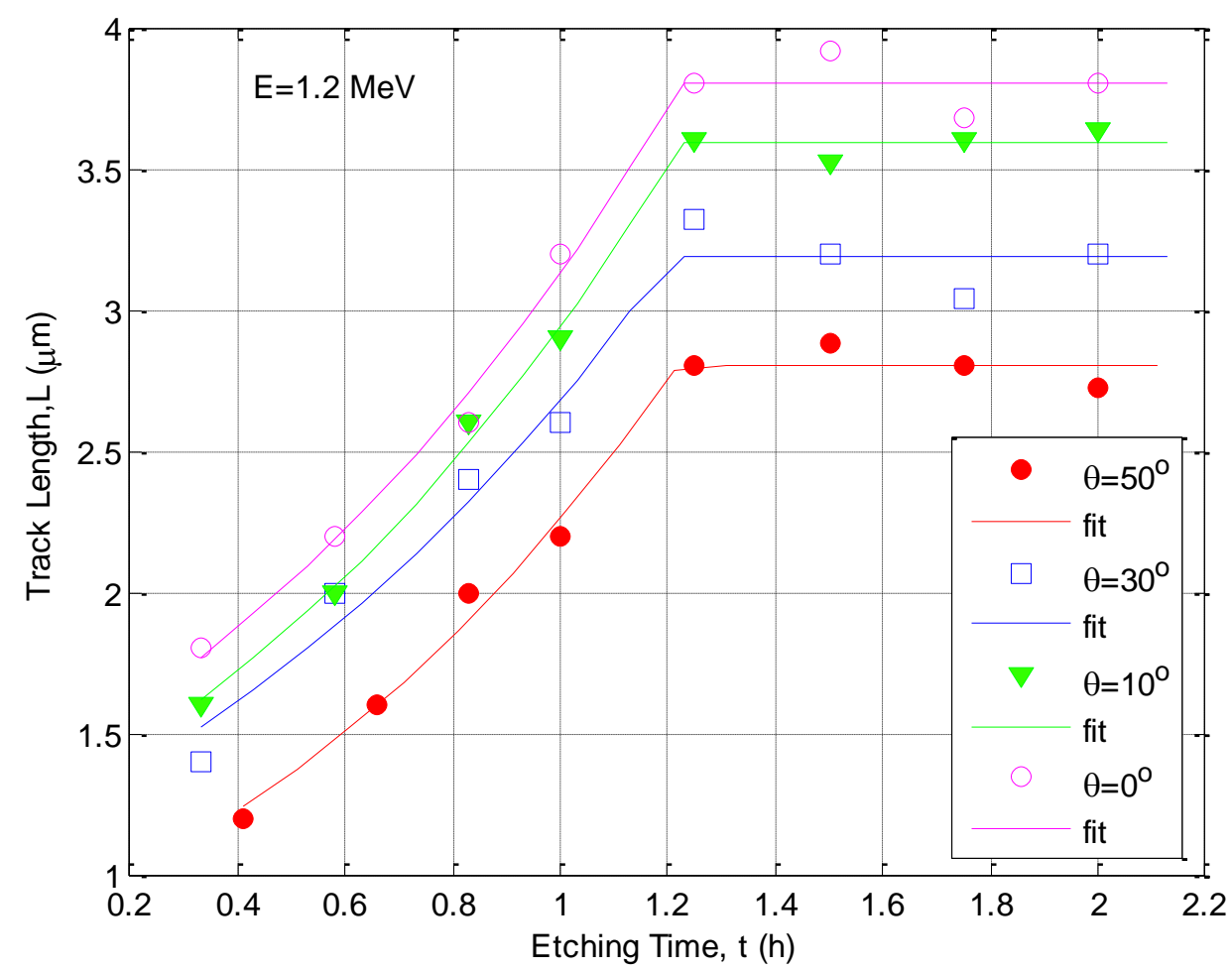

الثكل (4): طول الاثر دالة لزمن القشط للطاقة 1.2 MeV بزوايا سقوط مº, $10,30,50)$

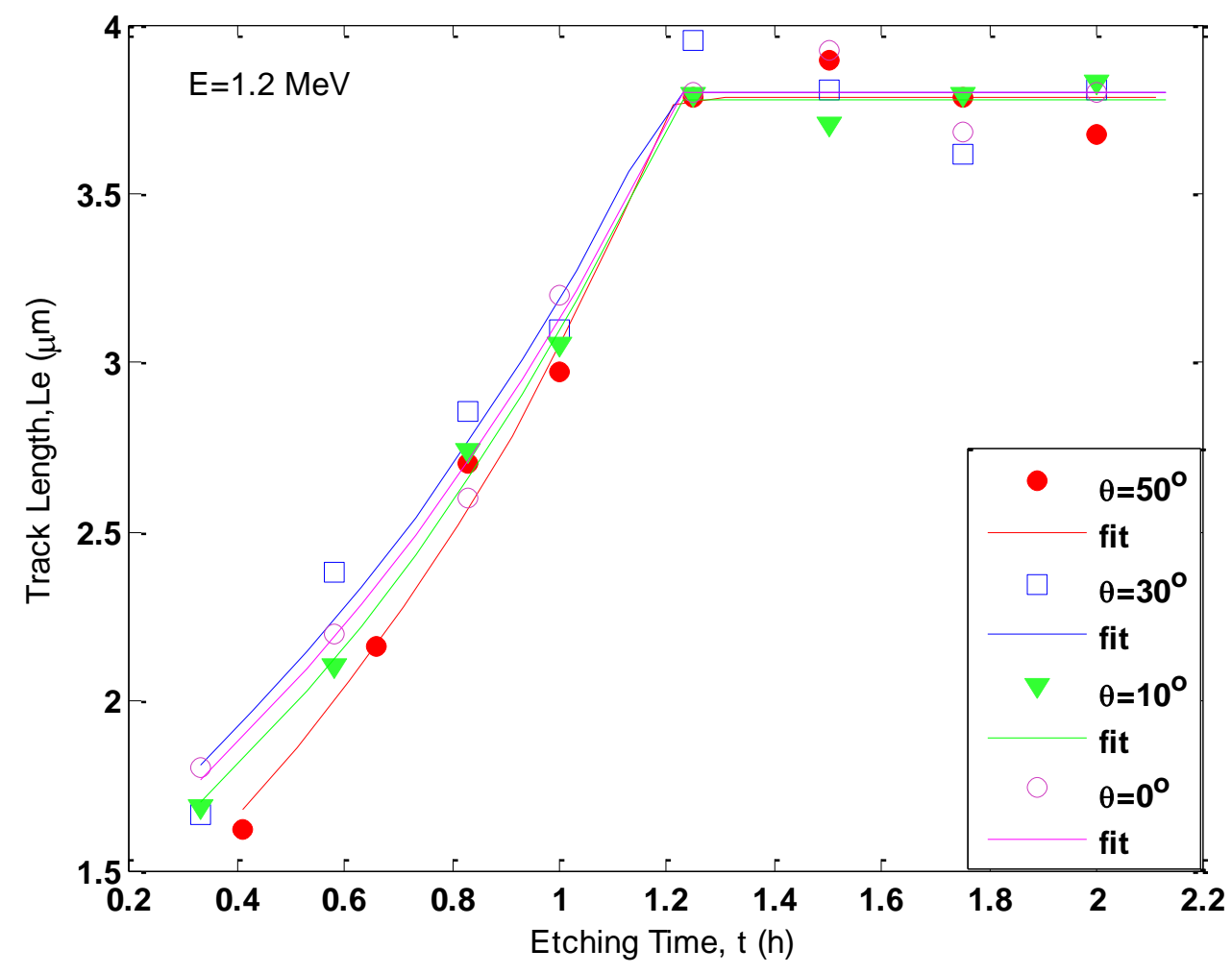

الثكل (5): طول الاثر دالة لزمن القشط للطاقة عند الاخذ بنظر الاعتبار زاوية السقوط 
ولقلة دراسات السقوط المائل فقد تم في هذا البحث دراسة معلمات الاثر في حالة السقوط المائل إذ حسب معدل قثط الاثر من المعادلة (2) دالة لزمن القشط كما هو مبين في الثكل (5) يبين ان معدل قثط الاثر لا يعتمد على زاوية السقوط وانما يكون له في اله

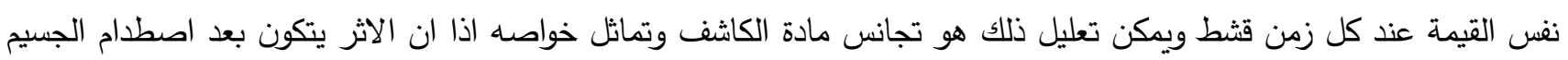

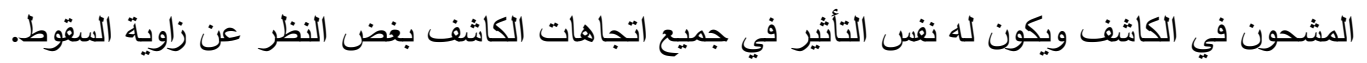

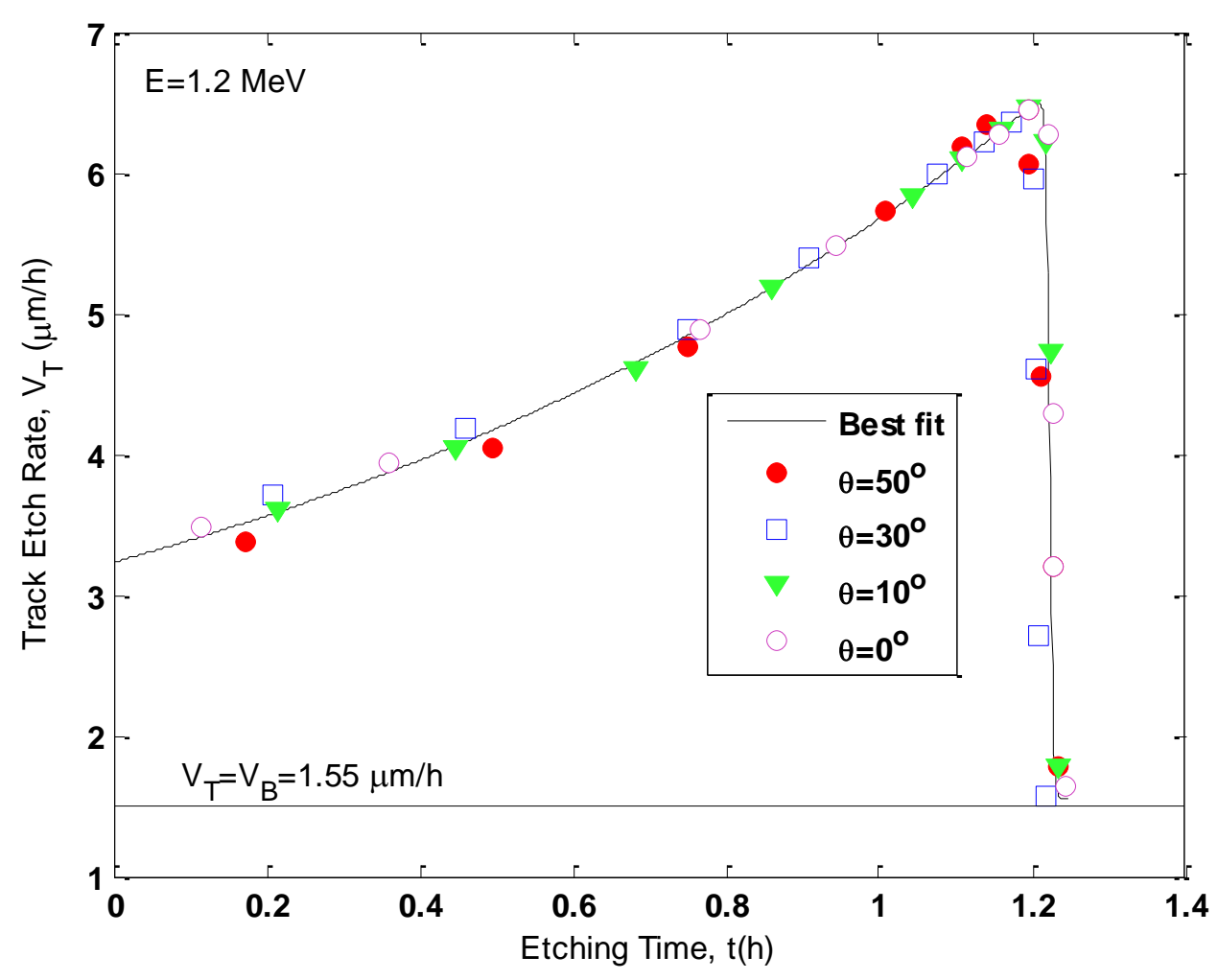

الشكل (6): معدل قشط الأثر دالة لزمن القشط لزوايا سقوط مختلفة.

من الثكلين (4,6 نلاحظ ان زمن ثبوت الاثر يكون نفسه تقريبا عند زوايا سقوط مختلفة وهذا منطقي كون معدل قثط الاثر يكون

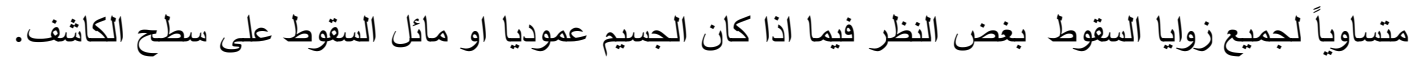
ولرسم العلاقة بين نسبة معدل القشط V دلة للمدى المتبقي $R^{\prime}$ الذي يمكن ايجاده من العلاقة التالية: يمثل مدى جسيمة الفا داخل الكاشف R= $\mathrm{L}_{\text {max }}+\mathrm{V}_{\mathrm{B}} \mathrm{t}_{\text {sat. }} R^{\prime}=R-x$ وصول طول الاثر الى اعظم قيمة و X=L-VB الثكل (7) يمثل العلاقة بين نسبة معدل القشط والمدى المتبقي يلاحظ من الثكل ان نسبة معدل القشط تزداد بشكل متسارع جدا في

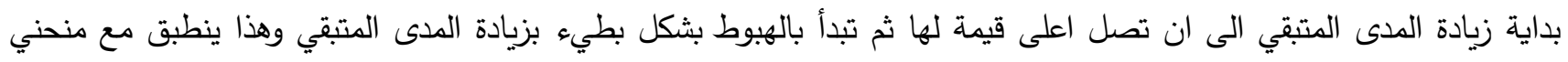

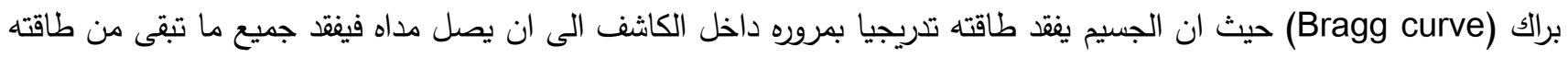
ويحدث اكثر ضرر في تلك المنطقة عندها تكون نسبة معدل القشط في قيمتها العظمى. 


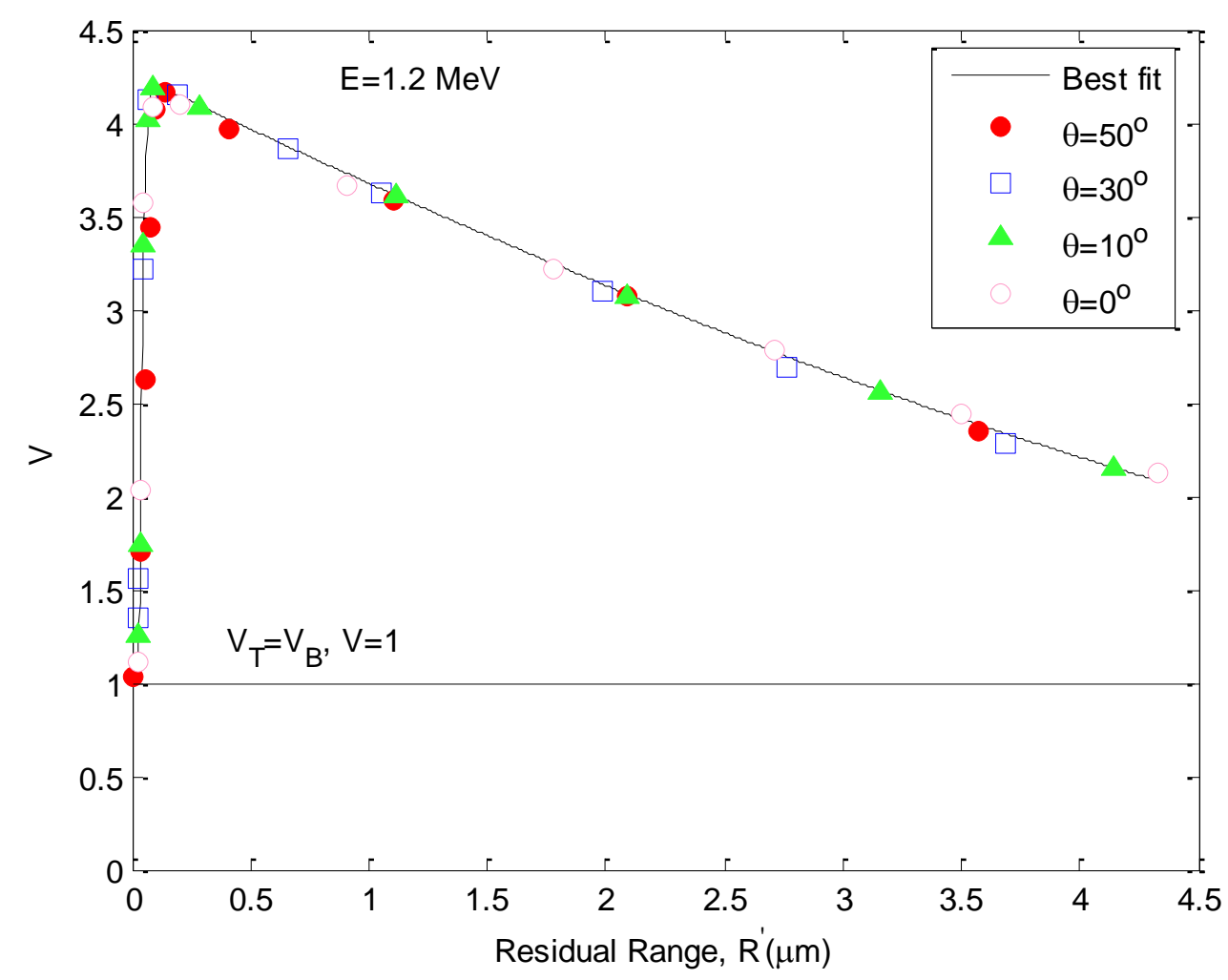

الثكل (7): نسبة معدل القشط دالة للمدى المتبقي لزوايا سقوط مختلفة.

ولأجل مقارنة اشكال الأثار التي حصلنا عليها تجريبيا استخدم برنامج Track-Test لرسم شكل الاثر نظريا حيث استخدمت المعادلة الاولى في البرنامج دعادلة Green et al, 1982 تحتوي هذه المعادلة على اربع معلمات افتراضية في البرنامج تم اخذها

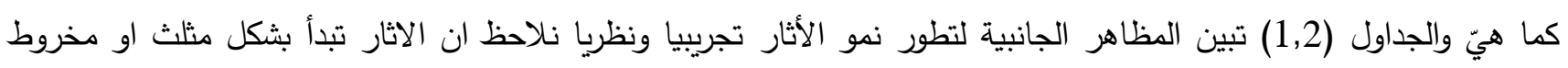

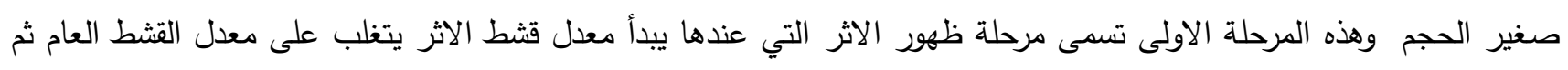
يستمر نموها الى ان تصبح مخروطية الثكل وهذه المرحلة هي الثانية والتي تم شرحها مسبقا وبتقدم عملية القشط تبدأ الاثار

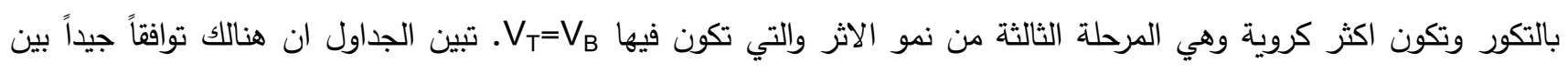

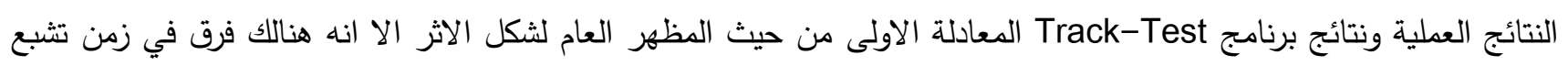
الأثر وطوله وهذا منطقي كون تلك النتائج اعتمدت على معلمات المعادلة نفسها. 
$\underline{\text { Journal of Education and Science (ISSN 1812-125X), Vol: 29, No: 3, } 2020(196-210)}$

الجدول (1): مقارنة بين النتائج العملية ونتائج برنامج Track -Test المعادلة الاولى عند الطاقة 1.2 MeV بزوايا سقوط $(\theta=0,10)^{\circ}$

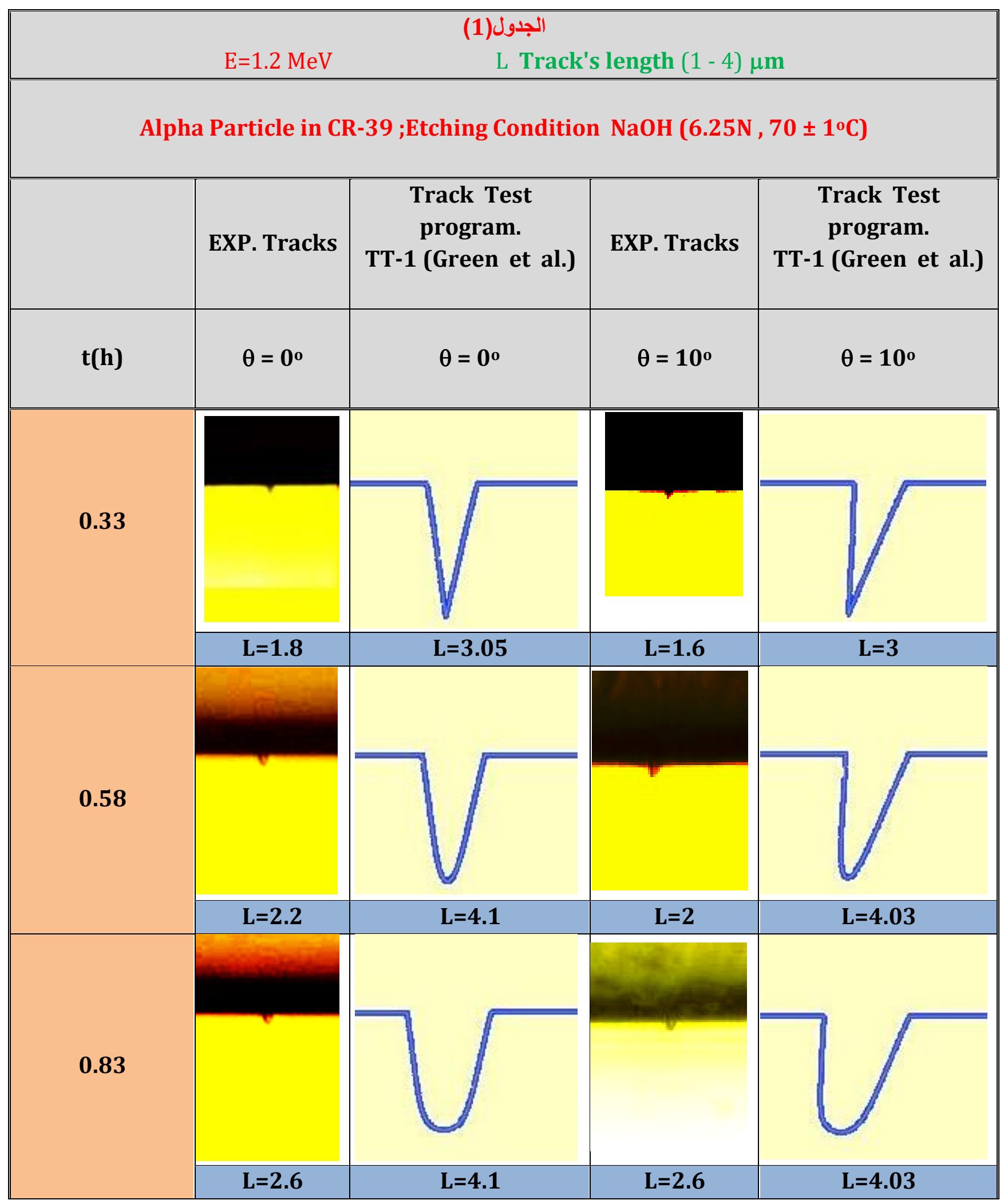


$\underline{\text { Journal of Education and Science (ISSN 1812-125X), Vol: 29, No: 3, } 2020(196-210)}$

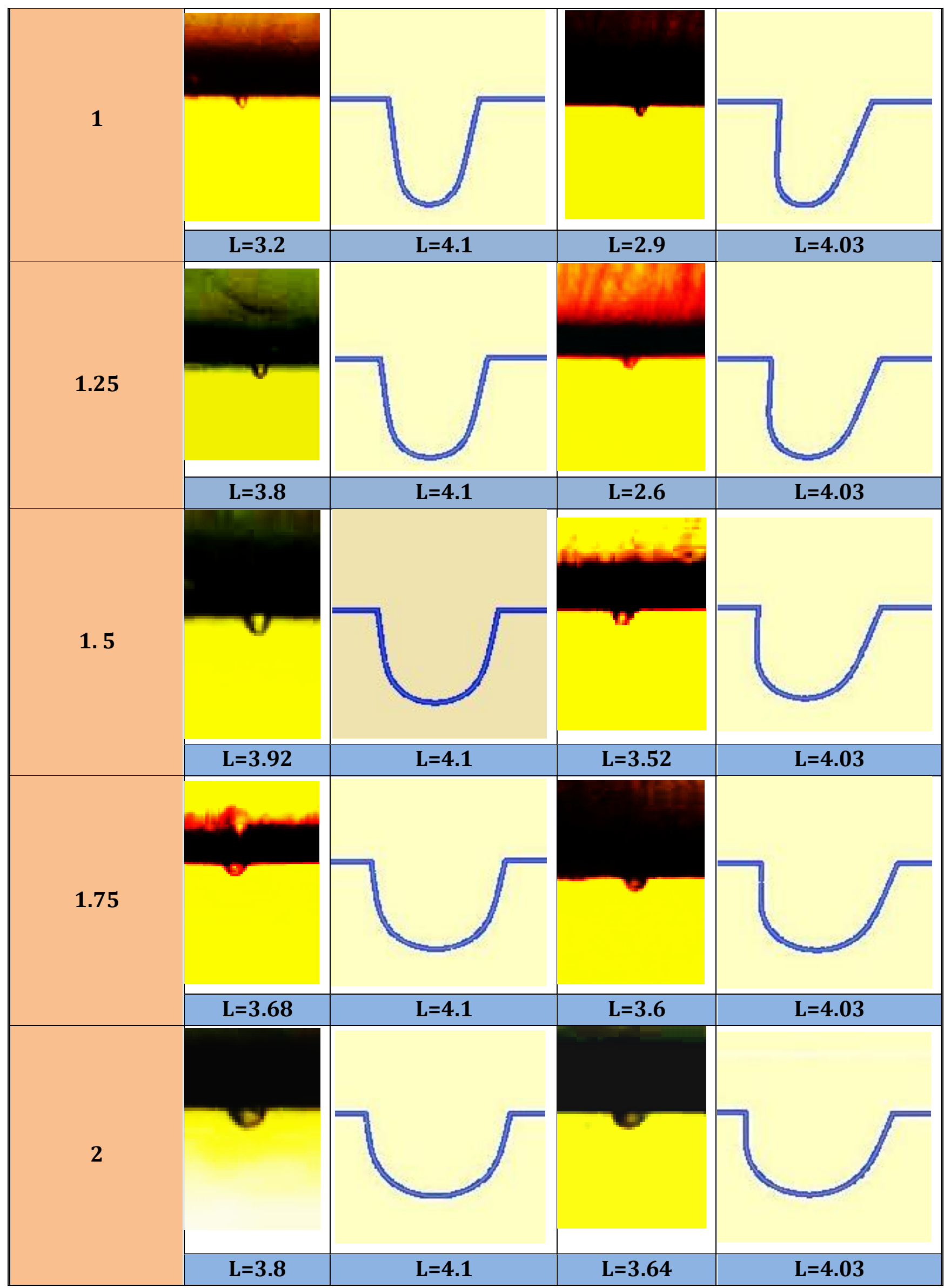


الجدول (2): مقارنة بين النتائج العملية ونتائج برنامج Track -Test المعادلة الاولى عند الطاقة 1.2 MeV بزوايا سقوط $(\theta=30,50)^{\circ}$

\begin{tabular}{|c|c|c|c|c|}
\hline \multicolumn{5}{|c|}{ (2) الجدول(2) } \\
\hline \multicolumn{5}{|c|}{ Alpha Particle in CR-39; Etching Condition $\mathrm{NaOH}\left(6.25 \mathrm{~N}, 70 \pm 1^{\circ} \mathrm{C}\right)$} \\
\hline & EXP. Tracks & $\begin{array}{c}\text { Track Test } \\
\text { program. } \\
\text { TT-1 (Green et al.) }\end{array}$ & EXP. Tracks & $\begin{array}{c}\text { Track Test } \\
\text { program. } \\
\text { TT-1 (Green et al.) }\end{array}$ \\
\hline $\mathbf{t}(\mathbf{h})$ & $\theta=30^{\circ}$ & $\theta=30^{\circ}$ & $\theta=50^{\circ}$ & $\theta=50^{\circ}$ \\
\hline \multicolumn{5}{|l|}{0.33} \\
\hline & $\mathrm{L}=\mathbf{1 . 4}$ & $L=2.58$ & $\mathrm{~L}=\mathbf{1 . 2}$ & $\mathbf{L}=2.15$ \\
\hline \multicolumn{5}{|l|}{0.58} \\
\hline & $\mathrm{L}=\mathbf{2}$ & $\mathrm{L}=3.44$ & $L=1.6$ & $\mathrm{~L}=2.34$ \\
\hline \multicolumn{5}{|l|}{0.83} \\
\hline & $\mathrm{L}=2.4$ & $\mathrm{~L}=3.44$ & $\mathbf{L}=\mathbf{2}$ & $L=2.34$ \\
\hline
\end{tabular}


$\underline{\text { Journal of Education and Science (ISSN 1812-125X), Vol: 29, No: 3, } 2020(196-210)}$

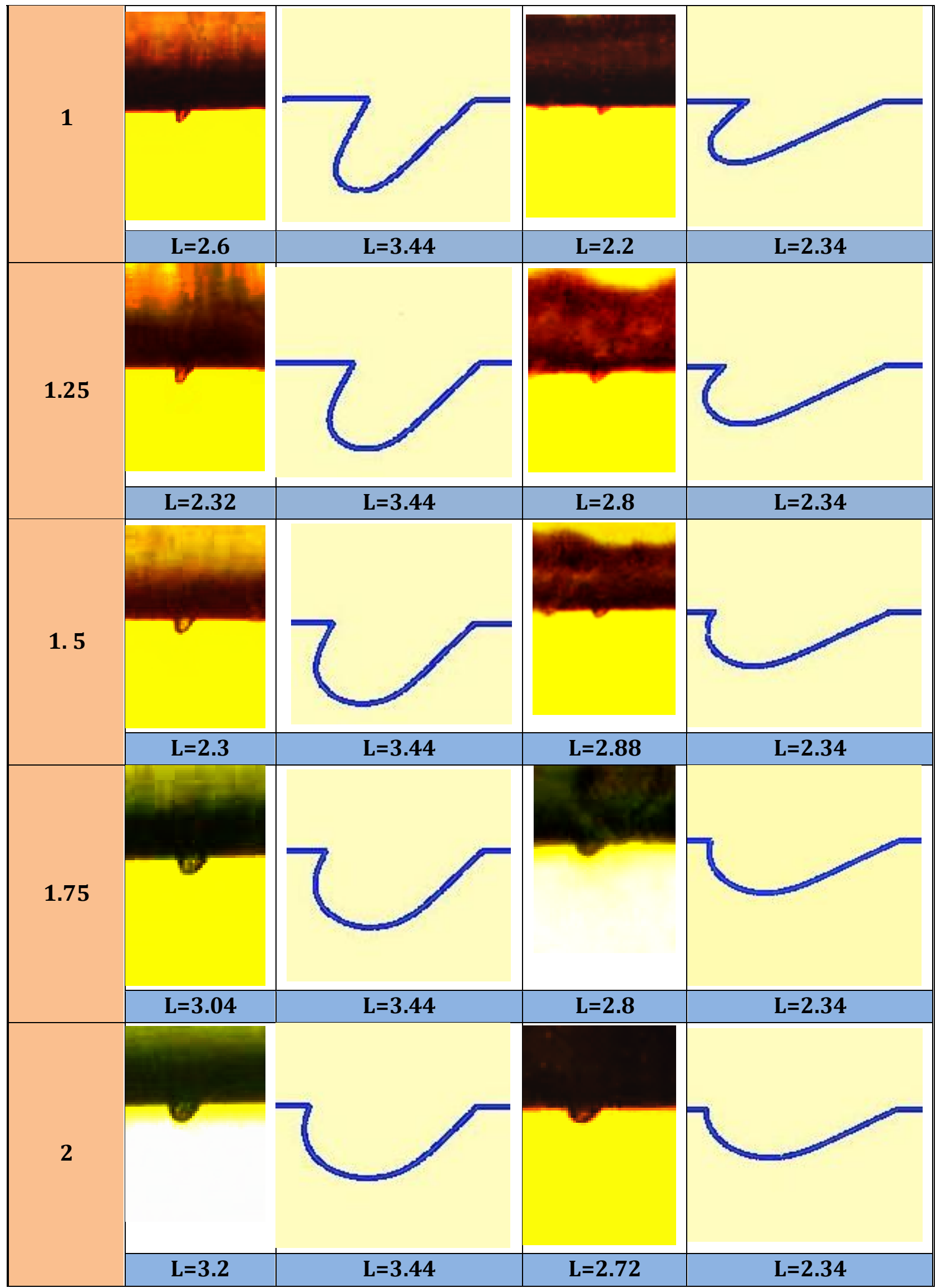


الثكل (8) يبين معدل نمو الاثر دالة للعمق (العمق العمودي) اذ نلاحظ من الشكل ان معدل نمو الأثر يكون اسرع بزيادة زاوية

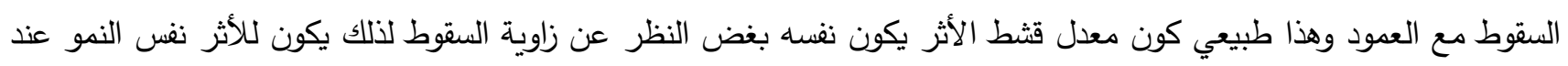

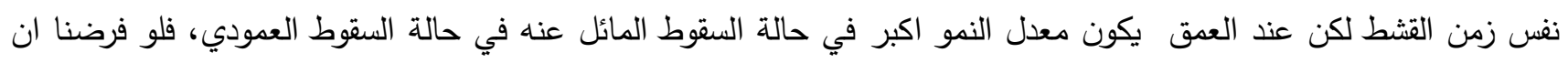
لدينا أثرين بنفس الطول احدهما عمودي والأخر مائل فعند قياس معدل نمو الأثر بالنسبة للعمق العمودي يتضح انه فيكون فئ اكبر كلما

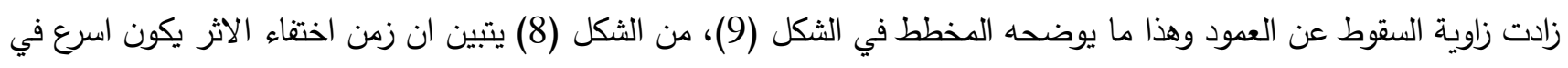

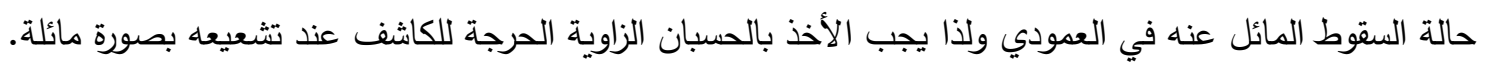

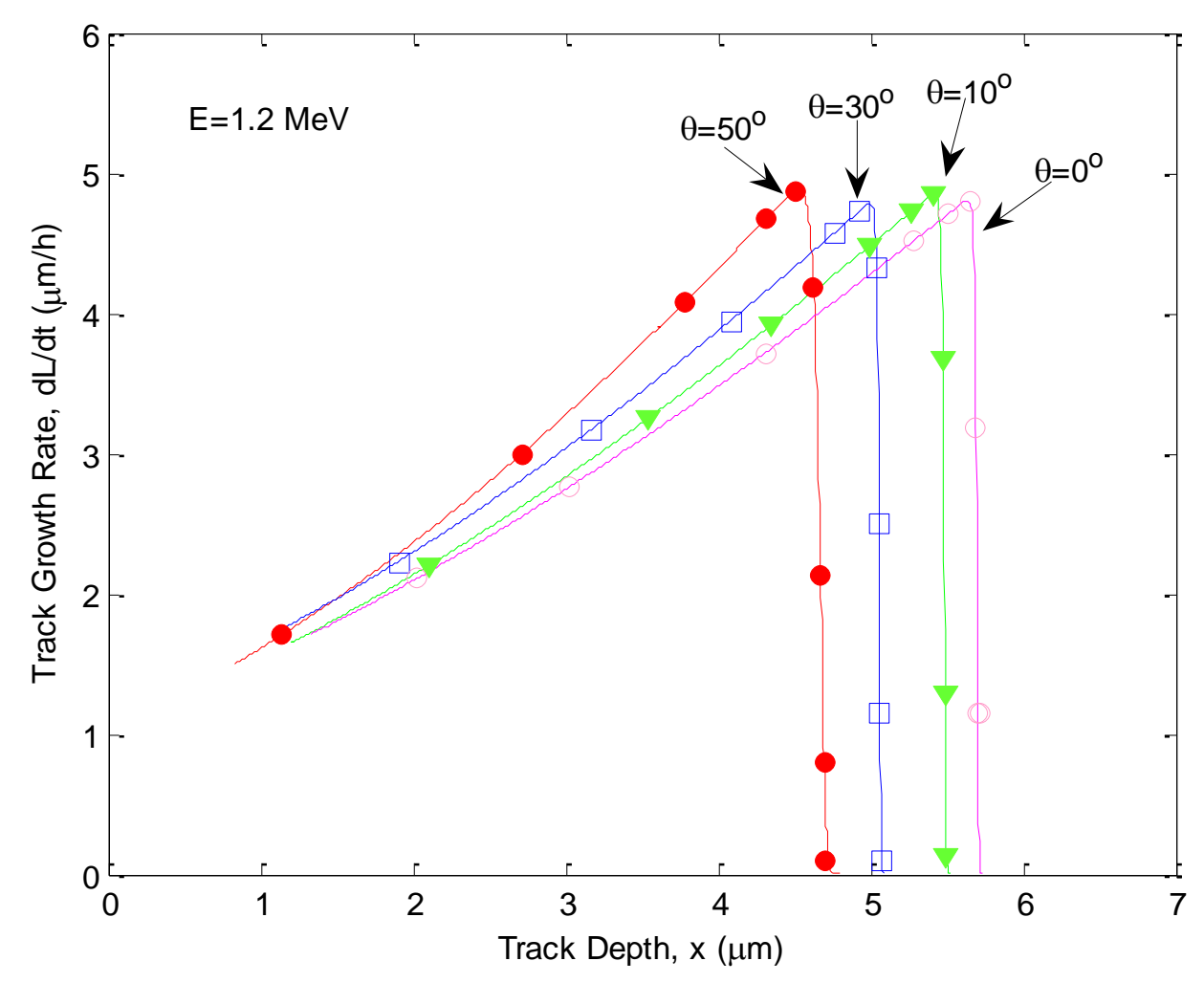

الشكل (8): معدل نمو الأثر دالة للعمق العمودي

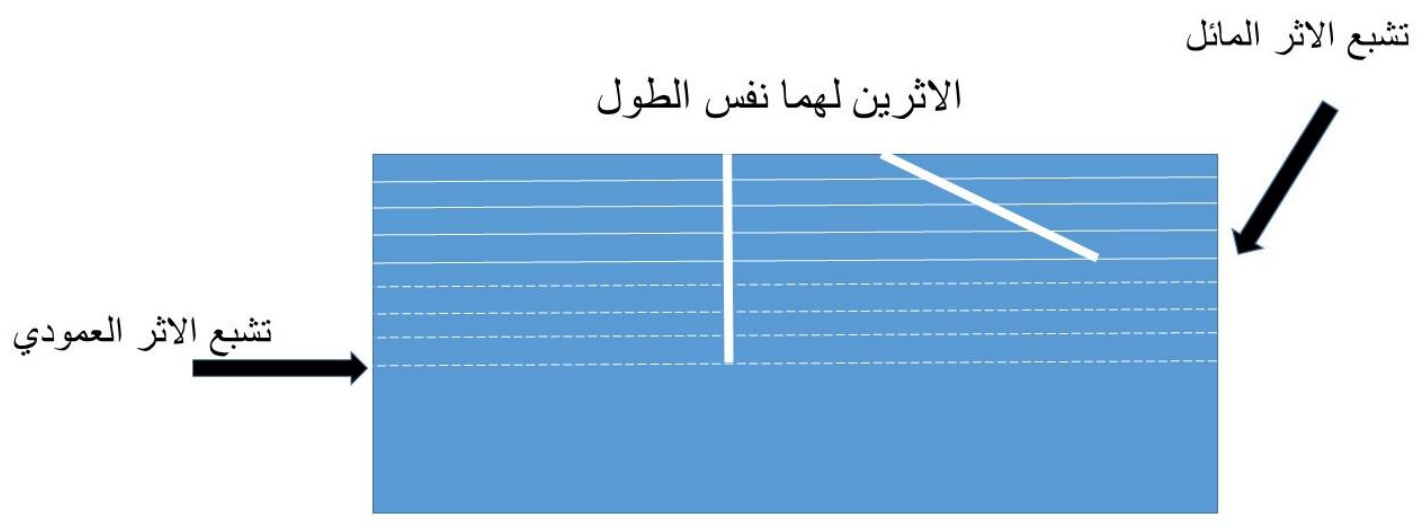

الثكل (9): أثران بنفس الطول احداهما مائل والأخر عمودي 
اشتملت الدراسة في هذا البحث على جزئين: الجزء الاول اشتمل على معرفة معدل القشط العام للكاشف VB باستخدام طريقة السمك

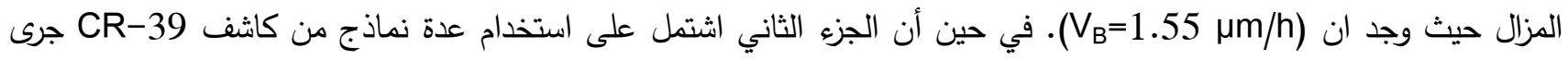

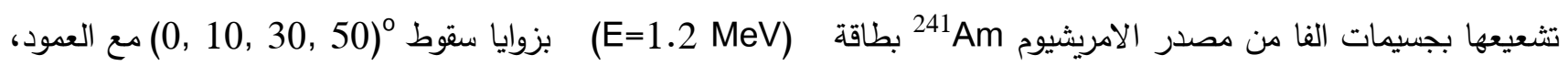

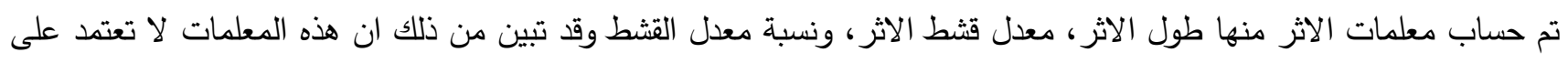
زاوية السقوط. دُرس معدل نمو الاثر دالة للعمق العمودي اذ تبين ان هذا النمو يزداد بصورة اسرع دالة للعمق العمودي كلما كانت

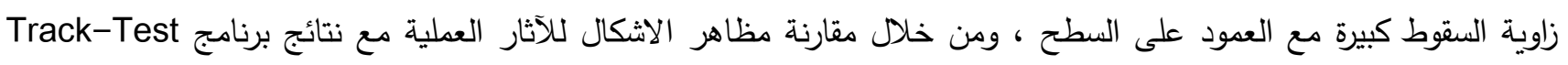

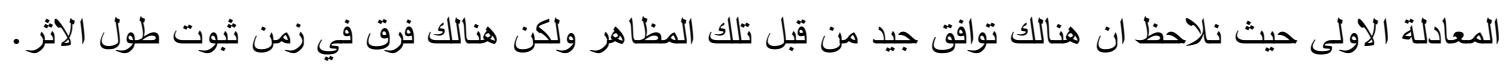

\section{Acknowledgements}

The authors is very grateful to the University of Mosul/ College of Education for Pure Sciences for their provided facilities, which helped to improve the quality of this work.

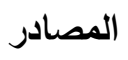

[1] Dörschel, B.; Hermsdorf, D.; Reichelt, U, Radiat. (2003) Meas. 37, pp. 563-571.

[2] Green, P. G.; Ramli, A. G.; Al-Najjar, S. A. R.; Abu-Jarad, F. and Durrani, S. A. and Durrani, S. A. (1982), Nucl. Instr. and Meth., 203, pp. 551-559.

[3] Dörschel, B.;Bretschneider, R ;Hermsdorf, D ;Kadner, K .and Kuhne, H .(1999), Radiat. Meas. 31, pp. 103-108.

[4] Al-Jubbori, Mushtaq Abed .(2017), Nuclear Inst. and Methods in phyics Research, A 871, pp. 54-58.

[5] Azooz, Aassim. A.; Al-Jubbori, Mushtaq. A .(2016), Applied Radiation and Isotopes.115, pp. 7480

[6] Al-Jubbori, Mushtaq Abed .(2016), Applied Radiation and Isotopes.118, pp. 228-231

[7] Azooz, A. A.; Hermsdorf, D.; Al-Jubbori, M.A .(2013), Radiat. Meas. 58, pp. 94-100.

[8] Azooz, A. A.; Al-Jubbori, M.A .(2013), Nuclear In struments and Methods in physics Research. B 316, pp. 171-175. 
[9] Azooz, A. A.; Al-Nia'emi, S.H.; Al-Jubbori, M.A .(2012), Computer Physics Communications, 183, pp. 2470-2479.

[10] Azooz, A. A.; Al-Nia'emi, S.H.; Al-Jubbori, M.A .(2012), Radiat. Meas. 47, pp. 67-72.

[11] Hermsdorf, D. (2009), Radiat. Meas. 44(3), pp. 283-288.

[12] Nikezic, D. and Yu, K.N. (2006), Computer Physics Communications, 174, pp. 160-165.

[13] Yu, K. N.; Ng, F. M. F. and Nikezic, D. (2005), Radiat. Meas. 40, PP. 380-383.

[14] Dörschel, B.; Hermsdorf, D.; Kadner, K. and Kühne, H. (1998), Radiat. Prot. Dosimetry. 78(3), PP. 205-212.

[15] Dörschel, B.; Hartmann, H. and Kadner, K. (1996), Radiat. Meas. 26(1), PP. 51-57.

[16] Fromm, M.;Membrey, F.; and Saouli, R .(1991), Nucl. Tracks Radiat. Meas. Vol. 19. Nos 1-4, pp, 163-168.

[17] Al-Jubbori M.A.D and Al-Shumaisy, S.A.M .(2015), Jordan Journal of Physics . 8(1), pp. 5767.

[18] Hermsdorf D and Reichelt U. (2010), Radiat. Meas. 45, 1000-1013 